\title{
Distribution Factor Method Modified for Determine of Load Contribute based on the Power Factor in Transmission Line
}

\author{
Syarifuddin Nojeng ${ }^{1}$, Syamsir ${ }^{2}$, Arif Jaya ${ }^{3}$, Andi Syarifuddin ${ }^{4}$, \\ Mohammad Yusri Hassan \\ ${ }^{1,2,3,4}$ Department of Electrical Engineering, Universitas Muslim Indonesia, Makassar, Indonesia \\ ${ }^{5}$ Centre of Electrical Energy Systems, Universiti Teknologi Malaysia, Johor, Malaysia
}

\begin{tabular}{l} 
Article Info \\
\hline Article history: \\
Received Mar 18, 2018 \\
Revised May 19, 2018 \\
Accepted Jun 2, 2018 \\
\hline Keywords: \\
Distribution factor method \\
Power factor \\
GLDF method \\
Transmission line usage
\end{tabular}

Transmission line usage

\begin{abstract}
This paper proposes a modification of distribution factor methods for identifying the load contribute in a transmission open access, with regard to the load power factor. This method may be considered as the first pricing strategy to be proposed in bilateral transaction for transmission usage, based on the actual use of the transmission network. The merit of this method relies on the existence of a load power factor with GLDF methods, which allocate the transmission cost, not only based on the amount of power flow but also on the load characteristic. A case study utilizing the IEEE 30-bus system was conducted to illustrate the contribution of the proposed method in allocating the transmission usage to the user in a fair manner.
\end{abstract}

Copyright $@ 2018$ Institute of Advanced Engineering and Science. All rights reserved.

\section{Corresponding Author:}

Syarifuddin Nojeng,

Department of Electrical Engineering,

Universitas Muslim Indonesia, Makassar, Indonesia.

E-mail: syarifuddin.nojeng@umi.ac.id

\section{INTRODUCTION}

The electric supply industry has been changing rapidly throughout the world as a consequence of the implementation of deregulation. In deregulation, the user of the interconnection network has to pay the cost of transmission service, which is determine of transmission line usage each user [1]. Many methods have been used or proposed to evaluate of the transmission usage allocation (TUA) through tracing method.

Bialek [2] proposed the principle of Proportional Sharing Method (PSM) to determine the contribution of each generator to the load. However, this method only considers positive contribution to the line flow. Meanwhile, Rudnick [3] proposed the method of tracking the power flow algorithm along with the Proportional Sharing principle by using a distribution method through the DC load flow model. Kirchen [4] proposed a tracking method using the principle of proportional assumption, which is a common method. This principle is based on the concept of grouping the buses and branches in the network system into homogeneous group. This method can also be used to calculate the losses contribution of a generator in the line. The disadvantages this method is that it cannot determine reverse power flow. F.Wu [5] developed a graph theory which can be used to calculate the contributions of each generator and loads to line flows, and also the real power transfer between individual generators and loads, while $\mathrm{Yu}$ [6] proposed a Tee Proportional Method (TPM) by using the principle of proportional development approach without using the inverse matrix. However, the method has not been tested for tracing reactive power.

Gubina [7] proposed a new method for determining the generator's contribution to a particular load, which uses the nodal generation distribution factors (NGDF-s). Pantos [8] recommended the modifications of the factors in determining GGDF to prevent expansion of the matrix, by using the load distribution factor (LDF) and the load distribution factor (LSF). This method can also be used to overcome the capacitive 
loading on the line. Valerie.S [9], on the other hand, proposed the concept of reference frame of the loop by modelling the network through injection of voltage and current at each node. All flow in the network is a representation of power delivery in a series of related loops. Hussain Shareef [10] suggested using modified nodal Equations to identify the real and reactive power transfer between generators and load.

Meanwhile, S.Abdulkader [11] recommended a tracking method based on the flow with the actual physical line, where the contribution of the division of the load current is in accordance with the crosssectional area of conductors. This concept is expressed by the components of a line into two parallel lines as equivalent line resistance. Ching [12] devised a method of transmission supplement charge allocation based on the topological analysis of power flows and DC linearized system model. This method is applied by assuming there is a line between the two ends of the shunt capacitor, which is exemplified as the fictitious load bus. Kaigue Xie [13] proposed an analytical model and algorithm based on the power flow, using Extended Incidence Matrix (EIM). In this method, firstly, a construction matrix EIM is defined as Matrix A, followed by defining the lossless power network (LPN) that provides the information about bus incidence relations and direction of active power in branch flow. Y.A. Altaturki [14] proposed the use of Current Adjustment Factor (CAF) method to allocate real and reactive power due to system linearity. In ref. [15] S. Nojeng, proposed determine of power flow based on the power factor aproach. The result of this method had proven its validity and consistency. However, this method failed to consider the power factor of the load, though it is one of the factors associated with the transmission transfer capability. In general, the power factor does not change the active power, but it can affect power quality.

The impact of the load characteristic (for instance; industries load, etc.) depends on the power flow contribution of each generator to the load in each transmission line. However, several methods have been used to the power flow tracing without the consideration of load characteristic.

This paper is organized as follows: Section II describes the current Distribution factor method, while Section III describes the proposed method based on the load power factor. Application of the proposed method on the IEEE-30 bus system and discussion are presented in Section IV. Finally, Section VI concludes this study paper.

\section{DISTRIBUTION FACTOR METHOD}

The Distribution factor uses the A factor to determine the power flow in each line, through a DC line flow approximation. This principle uses the topology factor distribution by implementing a matrix of Generation Shift Distribution Factor (GSDF), factors Generalize Generation Distribution Factor (GGDF) and Generalized Load Distribution Factor(GLDF) to determine the contribution of the generator or the load on each line.

$A$ Factors are defined as a sensitivity factor, calculated as follows:

$$
A_{i-j, k}=\frac{\left(z_{i k}-z_{j k}\right)}{x_{i j}}
$$

Matrix A also is defined as Generalization Shift Distribution Factor (GSDF).

Where:

$Z_{i k}=$ Reactance of line from bus i to user $\mathrm{k}$

$Z_{j k}=$ Reactance of line from bus $\mathrm{j}$ to user $\mathrm{k}$

$X_{i j}=$ Reactance of line from bus $\mathrm{i}$ to bus $\mathrm{j}$

For determining the load contribution to the line flow, the Generalized Load Distribution Factor (C factor) can be used, as follows:

$$
C_{(i-j, R)}=\frac{\left[F_{i-j}-\sum_{k=R} A_{i-j, k} \cdot L_{k}\right]}{\sum_{k} L_{k}}
$$

Equation (2) can also be defined as a $\mathrm{C}$ factor, where Fi-j is power flow in line i-j, Lk is load-k, $\mathrm{R}$ is Reference bus and k as bus-k. The Distribution Factor in the existing methods, expressed as Equation (1) to (2), only shows the contribution of the power flow to each generator and load based on the line impedance. This method, however, only considers the line parameters (line reactance), the power injected by the 
generators and the load consumption, without considering the characteristics of each load. In reality, it does not reflect the characteristics of the load (ie. load power factor) which can affect the transmission line usage.

\section{PROPOSED METHOD}

Poor power factor will cause a decrease in transmission power transfer capability. Figure 1 shows a single line diagram, with the resistance ignored.

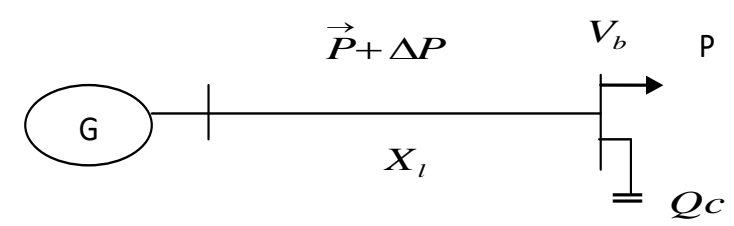

Figure 1. Single line diagram

The line impedance can be expressed as follows:

$$
Z_{l}=R_{l}+j X_{l}
$$

The real power $\mathrm{P}$ can be expressed as follows:

$$
P=V . I \cdot \cos \phi
$$

Where:

$=$ Angle betwen the reactive component with active componen in line where $\mathrm{V}$ and $\mathrm{I}$ are the voltage and $\phi$ current, respectively.

$Z_{l}=$ Line impedance

With assumption that $\mathrm{X} 1$ is line reactance of transmission line based on reference power factors, i.e (cos phi=0.8). Hence, by assuming $\mathrm{Rl}=0$, we can obtain:

$$
P=V^{2} \cdot X_{l} \cdot \cos \phi
$$

Assuming that the $\mathrm{Vb}$ (old) and $\mathrm{Vb}$ (new) are voltages in bus as constant before and after improving the load power factor, the following Equation is formulated:

$$
P_{\text {old }} X_{\text {old },} \approx P_{\text {new }} X_{l, \text { new }}
$$

Therefore:

$$
X_{l, \text { new }}=\frac{X_{\text {old }}}{\left[\frac{P_{\text {new }}}{P_{\text {old }}}\right]}
$$

With consideration of value from Figure 1, Equation 7 can be expressed as follows:

$$
X_{l, \text { new }}=\frac{X_{\text {old }}}{\left[\frac{P_{\text {old }}+\Delta P}{P_{\text {old }}}\right]}=\frac{X_{\text {old }}}{\left[1+\frac{\Delta P}{P_{\text {old }}}\right]}
$$

After eliminating the power flow in $P_{(\text {new }}$ and $P_{(o l d)}$, Equation 8 can be written as: 


$$
X_{l, \text { new }}=\frac{X_{l, \text { old }}}{\left[1+\left(\frac{\cos \phi_{\text {old }}-\cos \phi_{\text {new }}}{\cos \phi_{\text {old }}}\right)\right]}
$$

By substituting Equation (9) to (1), we can obtain a new A factor as follows:

$$
A_{i-j, k}(\text { new })=\frac{\left(z_{i k, \text { new }}-z_{j k, \text { new }}\right)}{x_{i j, \text { new }}}
$$

Meanwhile, the load contribution based on power factor for each line can be expressed as follows:

$$
C_{i-j, R}(\text { new })=\frac{F_{i-j_{(n e w)}}-\sum_{p=R} A_{i-j, k}(\text { new }) L_{k}}{\sum_{g} L_{k}}
$$

To illustrate how the modified distribution factors can be used to determine the contribution of the generator and load to the line flow, a 3 bus system was considered for application, as shown in Figure 2.

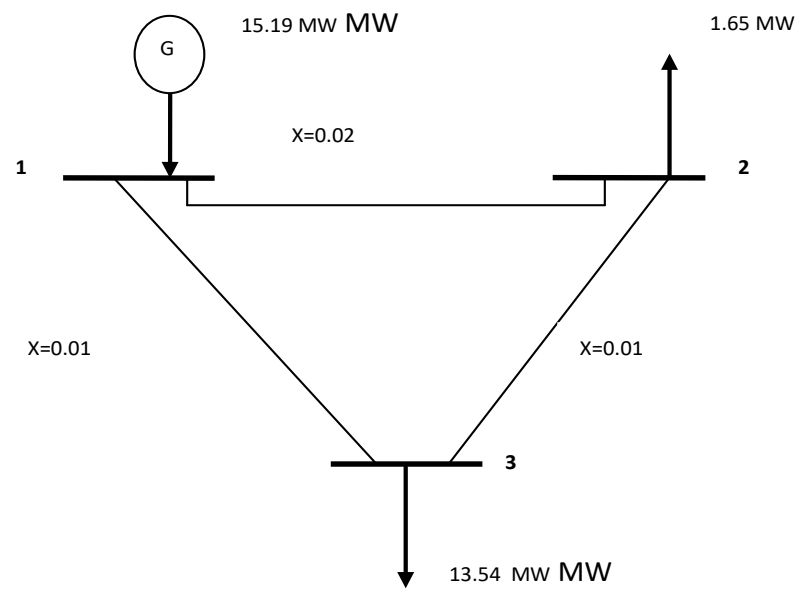

Figure 2. The 3 bus systems simple

Case: This method was verified for the 3 bus samples, which consisted of one generator (G1) and two load (L2 and L3). GSDF and modified GSDF were obtained by using Equation (10 ) and (11), as shown in Table 1. The GSDF was then compared with the proposed GGDF method, based on load characteristics. These load characteristics were estimated based on the power factor condition in load 2 (by assuming GSDF having a power factor 0.8 , taken as reference power factor).

Similarly, to obtain modified GLDF, Equation (10) and (11) had been used to obtain the original GLDF and modified GLDF, as shown in Table 3 (by assuming GLDF to have power factor of 0.8, taken as reference power factor).

Table 1. Comparison Between GSDF and Modified GSDF Based on the Power Factor in Load 2.

\begin{tabular}{ccc}
\hline & \multicolumn{2}{c}{ Modified GSDF } \\
GSDF existing & $\cos \Phi=0.85$ (lagging) & $\cos \Phi=0.90$ (lagging) \\
\hline $0-0.500-0.250$ & $0-0.515-0.274$ & $0-0.543-0.319$ \\
$0-0.500-0.750$ & $0-0.484-0.726$ & $0-0.456-0.680$ \\
$0-500-0.250$ & $00.484-0.274$ & $00.456-0.319$ \\
\hline
\end{tabular}


Table 2. Comparison Between GLDF and Modified GLDF Based on the Power Factor in Load 2

\begin{tabular}{ccc}
\hline \multirow{2}{*}{ GLDF existing } & \multicolumn{2}{c}{ Modified GLDF } \\
& $\cos \Phi=0.85$ lagging & $\cos \Phi=0.90$ lagging \\
\hline 00.5000 .250 & 00.5150 .274 & 0.5430 .319 \\
00.5000 .750 & 00.4840 .726 & 00.4560 .680 \\
$0-0.5000 .250$ & $0-0.4840 .274$ & $0-0.4560 .319$ \\
\hline
\end{tabular}

Table 3. Contribution of Load in Each Line

\begin{tabular}{ccccc}
\hline \multirow{2}{*}{ Load } & Line & \multicolumn{2}{c}{$\begin{array}{c}\text { Contribution of load in each line (MW) } \\
\text { GLDF }\end{array}$} & \multicolumn{2}{c}{$\begin{array}{c}\text { Modified GLDF } \\
\text { From To }\end{array}$} & $\begin{array}{c}\text { Method existing } \\
\text { L2 }\end{array}$ & $1-2$ & 0.825 & 0.845 & 0.896 \\
& $3-2$ & 0.825 & 0.805 & 0.754 \\
& Total & 1.650 & 1.650 & 1.650 \\
& $1-3$ & 10.155 & 9.830 & 9.214 \\
L3 & $2-3$ & 3.709 & 3.709 & 4.325 \\
& Total & 13.540 & 13.540 & 13.540 \\
\hline
\end{tabular}

\section{CASE STUDY}

In this case study, the proposed method was tested on the IEEE 30-bus system, as shown in Figure 3. The transmission network data used in the IEEE 30-bus system was referred from [14]. In this system, six generators were employed at buses 1, 2, 13, 22, 26 and 27, while 20 loads were located at buses $2,3,4,7,8,10,12,14,15,16,17,18,19,20,21,23,24,26,29$ and 30 . The generators served a total system load of $259 \mathrm{MW}$.

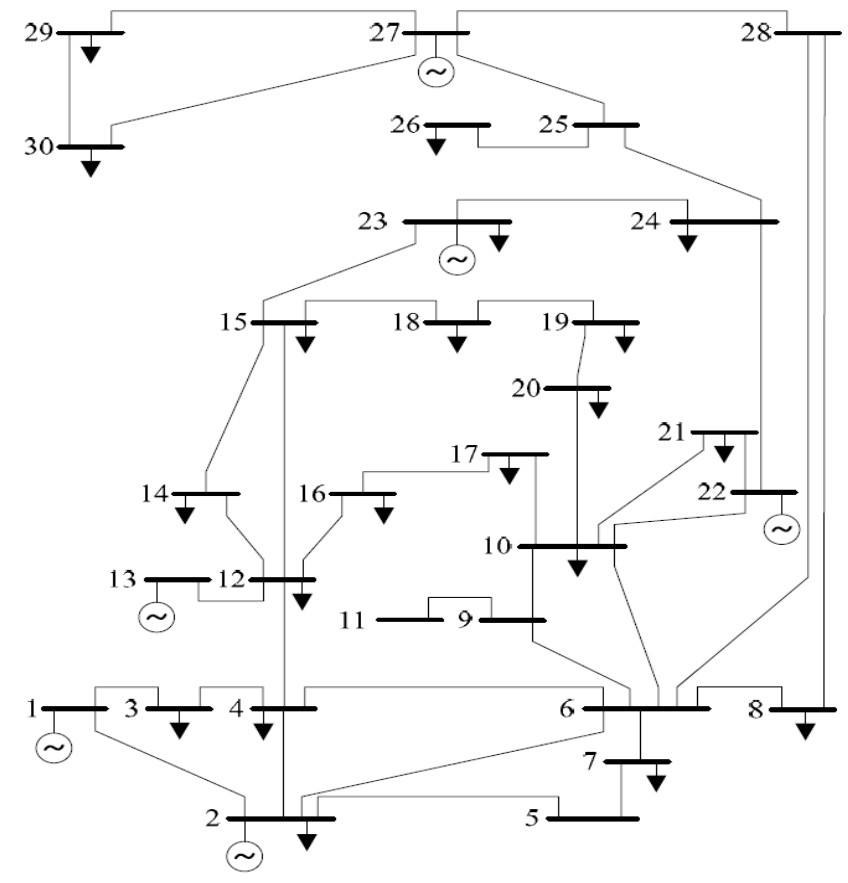

Figure 3. IEEE 30-bus system

Table 4 shows the contribution of each load in line, obtained by using modified Distribution Factor method. As can be seen from the results, both methods showed difference in the power flow in line. The GLDF modified showed that the load contribution in line was in accordance with the changes of the power factor at load L5. For instance, by using the original GLDF method, load L5, contributed 46.673 MW to line 1-2. On the other hand, by using the proposed method, the contributions of load L5 were 50.016 MW 
( $\cos \Phi=0.85$ lagging) and 55.462 ( $\cos \Phi=0.90$ lagging), respectively. As load L7 was related to bus 5 , there were also changes due to the connected line 5-7. Similarly, load L7 contributed 6.6675 MW $(\cos \Phi=0.85$ lagging) and 7.4923 MW ( $\cos \Phi=0.90$ lagging) to line 5-7. Both methods had the same total flow contribution of the lines related to the respective load. For example, the total flow contributed by L5 to line 2-5, and 7-5 for both methods was 94.2 MW. This happened similarly to L7 and L30, which produced 22.8 MW and 10.6 MW, respectively.

Table 4. Contribution of generator Using the GLDF and Modified GLDF Based on the Power Factor in Load 5

\begin{tabular}{|c|c|c|c|c|}
\hline \multicolumn{5}{|c|}{ Contribution of loads L5,L7 and L30 in each line (MW) } \\
\hline \multirow[t]{2}{*}{ Load } & \multirow{2}{*}{$\begin{array}{c}\text { Line } \\
\text { From- To }\end{array}$} & \multirow{2}{*}{$\begin{array}{l}\text { GLDF } \\
\text { method }\end{array}$} & \multicolumn{2}{|c|}{ Modified GLDF } \\
\hline & & & $\cos \Phi=0.85$ lagging & $\cos \Phi=0.90$ lagging \\
\hline \multirow[t]{3}{*}{ L5 } & $2-5$ & 48.67 & 50.02 & 55.46 \\
\hline & $7-5$ & 45.53 & 44.18 & 38.74 \\
\hline & Total & 94.20 & 94.20 & 94.20 \\
\hline \multirow[t]{3}{*}{ L7 } & $5-7$ & 6.07 & 6.67 & 7.49 \\
\hline & $6-7$ & 16.73 & 16.13 & 15.31 \\
\hline & Total & 22.8 & 22.80 & 22.80 \\
\hline \multirow[t]{3}{*}{ L30 } & $30-27$ & 6.24 & 6.24 & 6.24 \\
\hline & $30-29$ & 4.33 & 4.33 & 4.33 \\
\hline & Total & 10.67 & 10.67 & 10.67 \\
\hline
\end{tabular}

\section{CONCLUSION}

This paper has presented the ability of the modified Distribution Factors method for identifying the contribution of generator and load to transmission line flow, due to various load power factors. Determination of load contribution to electricity grid system has become important to the deregulated power system, especially for bilateral transaction in the electrical energy supply. By considering power factor variation from the users, the most efficient policy for determining the transmission charges for users (for instance: industry consumer) can be established. This case study results, based on IEEE 30-bus system, have shown that the proposed method can effectively determine the generator and load share in a fair manner.

\section{REFERENCES}

[1] Syarifuddin Nojeng, et.al. Harmonic Distortion Contribution for the Transmission Loss Allocation in Deregulated Energy Market: A New Scheme for Industry Supply. J.Elect.Engineering Technology, vol 10, no.1, 2015, : 1-7.

[2] Bialek, J. Tracing the flow of electricity. IEE Proceedings-Generation Transmission and Distribution,1996. 143(4) : 313-320.

[3] Hugh Rudnick, Manuel Soto. Rodrigo Palma. Use of system approaches for transmission open access pricing. Electrical Power and Energy Systems, 1999(21):125-135.

[4] D. Kirschen, R. Allan, and G. Strbac. Contributions of individual generators to loads and flows. IEEE Trans. Power System, vol. 12, $1997:$ 52-60.

[5] Felix F. Wu, Y. Ni, P. Wei.. Power transfer allocation for open access using graph theory - Fundamentals and applications in systems without loop flows. IEEE Trans.Power Systems, 2000, vol. 15, no. 3 : 923-929.

[6] Yu Xiao, Peng Wang. Tracing nodal market power using proportional three method", Power Systems Conference and Exposition, 2004. IEEE PES.

[7] Ferdinand Gubina, David Grgi and Ivo Bani. A Method for Determining the Generators' Share in a Consumer Load. IEEE Transaction on Power System, vol. 15, no. 4, $2000: 1376-1381$.

[8] Pantos, M., Verbic, G., \& Gubina.F. Modified topological generation and load distribution factors. IEEE Transactions on Power Systems, 20(4)2005: 1998-2005.

[9] Lim, V. S. C., McDonald, J. D. F., \& Saha, T. K. Development of a new loss allocation method for a hybrid electricity market using graph theory. Electric Power Systems Research, 2009.

[10] Shareef, Hussain et al. Real and Reactive Power Transfer Allocation Utilizing Modified Nodal Equations. International Journal of Emerging Electric Power Systems: Vol. 9: Issue 6.

[11] Abdelkader, S. Determining generators' contribution to loads and line flows \& losses considering loop flows. International Journal of Electrical Power \& Energy Systems, 30(6-7),2008 : 368-375.

[12] Ching-Tzong Su,Ji-Horng Liaw. Complex power flow tracing considering convection lines using nominal-T model. International Journal of Electrical Power \& Energy, Volume 29, Issue 1, 2007: 28-35.

[13] Kaigui Xie, Jia qi Zhou, Wenyuan Li. Analytical model and algorithm for tracing active power flow based on extended incidence matrix. Electric Power Systems Research, 79,2009: 399-405. 
[14] Alturki, Y.A. Towards reactive power markets. Generation, Transmission \& Distribution, IET, Volume: 2 , Issue: 4,2008 ,pp. $516-529$.

[15] Syarifuddin Nojeng et.al. Improving the MW Mile Method Using the Power Factor Approach for Pricing in the Transmissions Line Services. IEEE Transactions on Power System, vol. 29, no. 5, 2014 :2042-2048. 